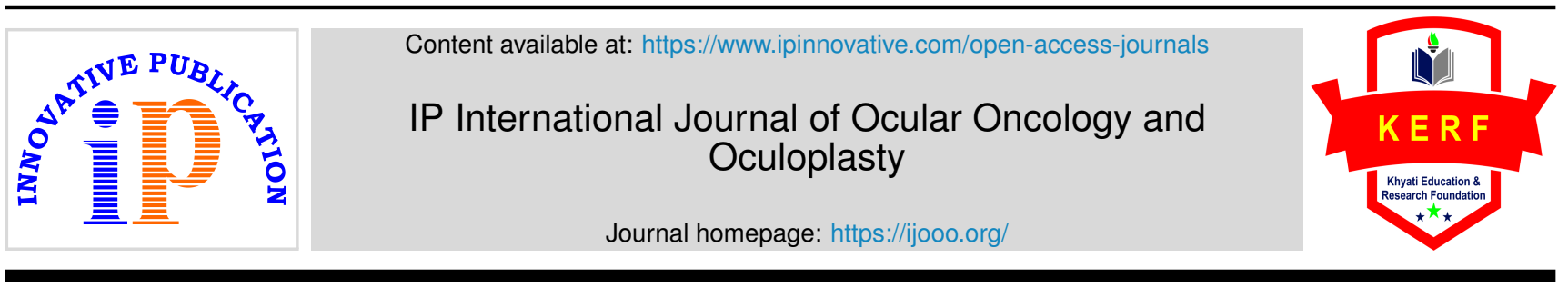

\title{
Editorial
}

\section{Indications for orbital exenteration in COVID-19 associated Rhino-orbito-cerebral Mucormycosis}

\author{
Rajendra Prakash Maurya ${ }^{1, *}$ \\ ${ }^{1}$ Dept. of Orbit, Ocular, Oncology and Oculoplasty, Unit Regional Institute of Ophthalmology, Institute of Medical Sciences, \\ Banaras Hindu University, Varanasi, Uttar Pradesh, India
}

\section{A R T I C L E I N F O}

Article history:

Received 08-06-2021

Accepted 12-06-2021

Available online 24-07-2021
(C) This is an open access article distributed under the terms of the Creative Commons Attribution License (https://creativecommons.org/licenses/by/4.0/) which permits unrestricted use, distribution, and reproduction in any medium, provided the original author and source are credited.
Rhino-orbito-cerebral Mucormycosis (ROCM) is an emerging, rapidly progressive life threatening opportunistic fungal infection caused by ubiquitous saprophytic fungi belonging to the class of zygomycetes, order Mucorales. It is the most common form of mucormycosis, associated with high mortality rate. The major risk factors for ROCM include immunosuppressive conditions, uncontrolled diabetes, ketoacidosis, neutropenia, renal failure, corticosteroid therapy, organ transplantation etc., Its incidence has significantly risen during COVID-19 pandemic (second wave) specially in patients with the above mentioned risk factors, moreover the inadequate lymphocyte count found in COVID-19 patients might be one of the main risk factors responsible for secondary fungal invasion. ${ }^{1}$

This fungi has a tendency to invade the arterial wall which leads to thrombosis consequently leading to ischemia and tissue necrosis. ${ }^{2}$ Mucormycosis mainly originates in the nasal or oral mucosa and then spreads to paranasal sinuses and enters the orbital cavity by bony destruction or via nasolacrimal ducts, lymphatics and neurovascular bundles 3 it can lead to intracranial or cavernous sinus invasion as well which occurs either through the orbital apex or through pterygopalatine fossa.

\footnotetext{
* Corresponding author.

E-mail address: mauryarp_bhu@yahoo.com (R. P. Maurya).
}

The early diagnosis of ROCM is often difficult and challenging due to its highly variable clinical presentation. ${ }^{4,5}$ The initial nonspecific clinical presentations are in the form of headache, fever, nasal congestion and discharge, epistaxis, mucosal necrosis and facial or dental pain. Ophthalmic manifestations gradually develop as infection spreads to the orbit. Oculo-orbital manifestations are mainly due to necrosis of cranial nerves (orbital apex syndrome), orbital cellulitis leading to orbital compartment syndrome and rarely intraocular invasion (endophthalmitis). ${ }^{6,7}$ Ophthalmic signs and symptoms of ROCM are highly variable. Common ocular findings are pain in the eyes, conjunctival hyperemia, chemosis, periorbital swelling, ptosis, proptosis, diplopia, ophthalmoplegia and vision loss. ${ }^{8,9}$ Sudden visual loss may be due to central retinal artery or ophthalmic artery occlusion, optic neuritis, optic nerve infarction or direct infiltration of the optic nerve, thrombosis or invasion of the cavernous sinus and rarely endophthalmitis. ${ }^{6,9,10}$ The unilateral cranial nerve palsy (III,IV,VI nerve), signs of retinal \& choroidal ischemia and optic nerve dysfunction are signs of orbital apex syndrome.

Common diagnostic tools are nasal endoscopy, contrast enhanced MRI (preferred) or CT Scan of PNS, orbit and brain. The definitive diagnosis of ROCM is based on histopathological identification of fungal structures, positive identification for Mucorales. The real-time PCR 
and DNA sequencing are commonly used molecular tools for identification of ROCM. ${ }^{11}$ Contrast enhanced MRI is the most preferred radio-imaging for ROCM. A characteristic and early radio-imaging finding of ROCM is Black Turbinate sign (non-enhancement of nasal mucosa over turbinate on post contrast $\mathrm{T}_{1} \mathrm{~W}$ weighted image). The signs of early orbital infection are retro-orbital fat stranding and edema of extraocular muscle (best seen in saturated $\mathrm{T}_{1} \mathrm{~W}$ sequence). The lateral displacement (abscess in medial orbit) and thickening of medial rectus muscle (due to muscle infiltration) are also features of early orbital infection. ${ }^{3}$ The optic nerve thickening and perineural enhancement with high signal intensity on diffusion -weighted imaging is indicative of optic nerve invasion. Isolated optic nerve involvement is suggestive of spread of infection through branches of ophthalmic artery and is an indication for early exenteration. ${ }^{3,12}$ In advanced stage orbital mucormycosis radiological findings are characterized by severe proptosis and tenting of the globe (posterior globe angle $<130$ due to optic nerve stretching) with non-enhancement of orbital fat and muscles which suggest marked retrobulbar necrotic soft tissue (Figure 1). In $\mathrm{T}_{2} \mathrm{~W}$ MRI imaging presence of soft tissue enhancement at the orbital apex with involvement of optic canal and superior orbital fissure (SOF) are highly suggestive of the orbital apex syndrome and heterogenous soft tissue enhancement from SOF to cavernous sinus with loss of concavity of cavernous sinus are radiological findings of cavernous sinus syndrome / thrombosis.

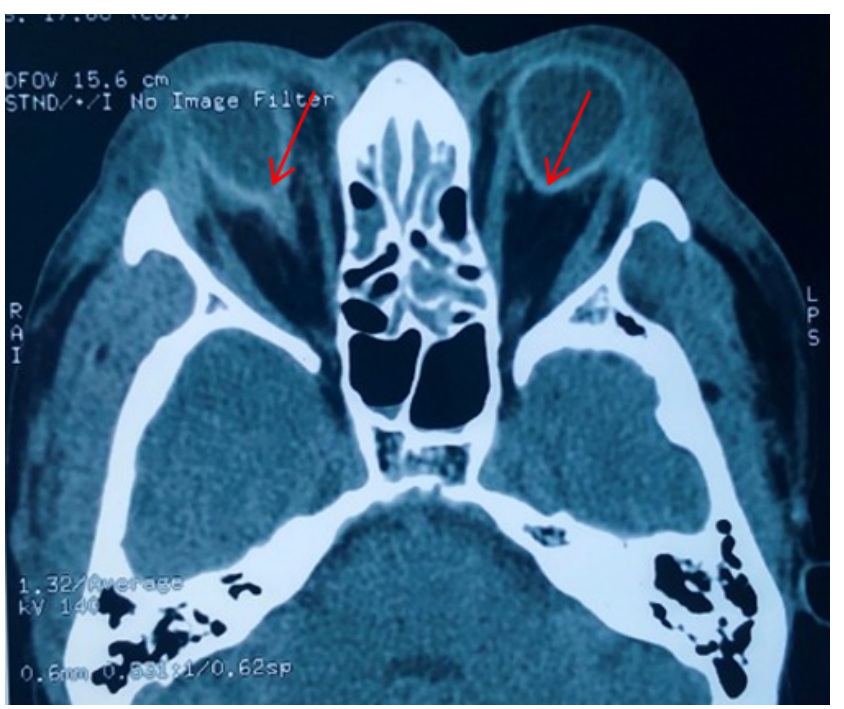

Fig. 1: CT Scan Axial view showing bilateral globe tenting.

On the basis of clinical and radiological findings orbital mucormycosis can be divided as mild or limited orbital disease and moderate to severe orbital mucormycosis. The limited orbital disease is characterized by no resistance on retropulsion of the globe, presence of extraocular movement $\&$ vision and orbital-imaging suggestive of mild disease (fat stranding or opacification in 1-2 quadrants of orbit). While features of moderate or severe disease are firm to hard consistency on retropulsion of the globe, restricted extraocular movements or frozen globe, loss of vision, ophthalmoplegia and radio-imaging suggestive of extensive orbital involvement. Honavar $\mathrm{SG}^{13}$ proposed a staging system, on the basis of clinico-radiological findings and results of nasal endoscopy to determine the extent and severity of ROCM. It divides ROCM in 4 stages; Stage I (disease limited to nasal mucosa), Stage II (involving paranasal sinuses), Stage III (involving the orbit) and Stage IV (CNS involvement).

The treatment of ROCM requires an individualized multidisciplinary, multimodal approach including combination of appropriate systemic antifungal treatment, surgical debridement of necrotic tissues and correction of underlying conditions like hyperglycemia and acidosis etc. The poor prognostic factors in ROCM are (i) delayed diagnosis and late initiation of appropriate treatment (ii) bilateral sinus involvement (iii) cerebral involvement (iv) hemiparesis (v) facial or periocular gangrene etc. ${ }^{6}$

As ROCM is a rapidly progressive fatal disease, it requires early and an aggressive surgical debridement of infected \& necrotic tissue of the paranasal sinuses and retro-orbital space which is recommended to obtain local control and to improve prognosis and survival rate. ${ }^{14}$ Repeated surgical debridement may be required to remove the infected tissue. The surgical debridement not only decreases the fungal load but also improves the response of systemic antifungal treatment, as delivery of drugs to infected / necrotic tissue is hampered due to vascular tropism.

Sometime more aggressive orbital surgery such as orbital exenteration may be required to decrease fungal load and prevent direct intracranial extension. ${ }^{15,16}$ Orbital exenteration is always associated with oculofacial disfigurement and psychological distress. Although orbital exenteration is a life saving procedure but unfortunately indications for doing exenteration remain unclear. ${ }^{17}$ The standard guideline for orbital exenteration in treating ROCM is not available in literature. ${ }^{18-20}$ Singh VP et al. suggested that orbital exenteration should be individualized based on retinal artery involvement, aggressiveness of the disease, underlying debilitating diseases, response of antifungal chemotherapy and visual status. ${ }^{20}$ Levinsen et al. recommended aggressive orbital exenteration when dealing a case of ROCM with orbital apex syndrome, peribulbar or facial necrosis with or without cranial nerve involvement. ${ }^{21}$

In case of limited orbital disease with predominant sinonasal involvement, extensive debridement of paranasal sinuses, medial orbital compartment with or without medial wall resection with retrobulbar injection of amphotericin$\mathrm{B}$ are recommended while for severe orbital disease early orbital exenteration with aggressive sino-nasal debridement 


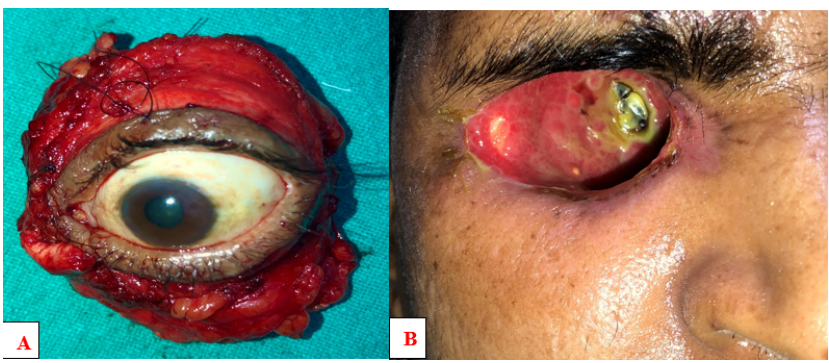

Fig. 2: (A) Specimen after orbital exenteration, (B) Photograph of post exenteration orbital socket.

has been recommended. 3,22 Shah $\mathrm{K}$ et al proposed "Sion hospital scoring system' to solve the dilemma associated with orbital exenteration in ROCM. The scoring system is based on three criteria; (1) clinical signs \& symptom, (2) direct \& indirect ophthalmoscopy and (3) radio-imaging. According to the score 1 point was given for mild symptoms/signs, 2-points for moderate symptoms/ signs and 3 points for severe symptoms \& signs. According to this scoring system patients having total score $\geq 23$ is said to be eligible for orbital exenteration. ${ }^{23}$

Hanover $\mathrm{SG}^{13}$ proposed management algorithm for ROCM in the setting of COVID-19, according to it orbital exenteration is recommended for extensive orbital involvement (stage 3c-d; central retinal artery or ophthalmic artery occlusion, superior ophthalmic vein thrombosis, orbital apex involvement, loss of vision and bilateral involvement), limited CNS involvement (stage 4c-d; focal or diffuse cavernous sinus involvement or thrombosis) and in case of extensive CNS involvement (stage 4c$\mathrm{d}$; involvement beyond cavernous sinus, skull base involvement, brain infarction or diffuse CNS involvement) if general systemic condition of patient permits surgery.

Finally we can say that the orbital exenteration is a life saving surgical modality of treatment and should be considered for ROCM patients having severe orbital \& CNS disease manifested as highly inflamed orbit with painful blind eye and frozen eye ball.

\section{Conflict of Interest}

None.

\section{References}

1. Gangneux JP, Bougnoux ME, Dannaoui E, Cornet $M$, Zahar JR. Invasive fungal diseases during COVID-19: We should be prepared. J Mycol Med . 2020;30(2):100971. do1:10.1016/].mycmed.2020.100971.

2. Joos ZP, Patel BC. Intraorbital irrigation of amphotericin B in the treatment of rhino-orbital mucormycosis. Ophthal Plast Reconstr Surg 2017;33(1):13-6.

3. Dave TV, Sreshta K, Varma DR, Nair AG, Bothra N, Naik $\mathrm{MN}$, et al. Magnetic resonance imaging in rhino-orbitalcerebral mucormycosis. Indian J Ophthalmol. 2021;69(7):1915-27. do1:10.4103/110.110 143921
4. Peterson KL, Wang M, Canalis RF, Abemayor E. Rhinocerebral Mucormycosis: Evolution of the Disease and Treatment Options. Laryngoscope. 1997;107(7):855-62. 101:10.109700005537109707000-00004

5. Lee DH, Yoon TM, Lee JK. Invasive fungal sinusitis of the sphenoid sinus. Clin Exp Otorhinolaryngol. 2014;7:181-7.

6. Yohai RA, Bullock JD, Aziz AA, Markert RJ. Survival factors in rhino-orbital-cerebral mucormycosis. Surv Ophthalmol 1994;39(1):3-22. doi:10.1016/s0039-6257(05)80041-4.

7. Ferry AP, Abedi S. Diagnosis and Management of RhinoOrbitocerebral Mucormycosis (Phycomycosis). Ophthalmology. 1983;90(9):1096-104. doi:10.1016/s0161-6420(83)80052-9.

8. Gamaletsou MN, Sipsas NV, Roilides E, Walsh TJ. Rhino-OrbitalCerebral Mucormycosis. Curr Infct Dis Rep. 2012;14(4):423-34.

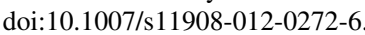

9. Bhansali A. Presentation and outcome of rhino-orbital-cerebral mucormycosis in patients with diabetes. Postgraduate Med J. 2004;80(949):670-4. doi:10.1136/pgmj.2003.016030.

10. Bhansali A, Sharma A, Gupta A. Orbital mucormycosis with endophthalmitis. Acta Ophthalmol. 2001;79:88-90.

11. Levinsen M, Kiilgaard JF, Thomsen C, Heegaard S, Nissen KR. Medical and surgical treatment of rhino-orbital-cerebral mucormycosis in a child with leukemia. Am J Ophthalmol Case Rep. 2021;22:101092. doi:10.1016/j.ajoc.2021.101092

12. Alsuhaibani AH, Badr FA, Al-Thubaiti G. Optic nerve thickening and infarction as the first evidence of orbital involvement with mucormycosis. Middle East Afr J Ophthalmol . 2012;19(3):340-2. 101:0.4103/0974-9233.97957

13. Honavar SG. Rhino-orbito-cerebral mucormycosis-Guidelines for diagnosis, staging and management. Indian J Ophthalmol. 2021;69:1361-5.

14. Vironneau P, Kania R, Herman P, Morizot G, Garcia-Hermoso $\mathrm{D}$, Lortholary $\mathrm{O}$, et al. Local control of rhino-orbito-cerebral mucormycosis dramatically impacts survival. Clin Microbiol Infect

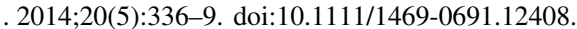

15. Uğurlu ŞK, Selim S, Kopar A, Songu M. Rhino-orbital Mucormycosis: Clinical Findings and Treatment Outcomes of Four Cases. Türk Oftalmoloji Dergisi. 2015;45(4):169-74. doi: $10.4274 / \mathrm{tj} 0.82474$

16. Jumaily M, Faraji F, Brunworth JD. Endoscopic orbital exenteration in the treatment of acute invasive fungal sinusitis. Acta Oto-Laryngol Case Repo. 2016;1(1):58-62. ब01:10.1080/23/72484.2016. 1220234.

17. Hernández OP, Calleros HP, Daguerre GM, González AS. RhinoOrbito-Cerebral Mucormycosis. Management Strategies to Avoid or Limit Intracraneal Affection and Improve Survival. Acta Otolaryngol Esp. 2015;66(6):348-52. doi:10.1016/j.otoeng.2015.01.002.

18. Hargrove RN, Wesley RE, Klippenstein KA, Fleming JC, Haik BG. Indications for Orbital Exenteration in Mucormycosis. Ophthal Plast Reconstr Surg. 2006;22(4):28691. ब01:10.1097/01.10p.0000225418.50441.ee

19. Colon-Acevedo B, Kumar J, Richard MJ, Woodward JA. The Role of Adjunctive Therapies in the Management of Invasive SinoOrbital Infection. Ophthal Plast Reconstr Surg. 2015;31(5):401-5. doi:10.1097/10p.000000000000488.

20. Singh VP, Bansal C, Kaintura M. Sinonasal Mucormycosis: A to Z. Indian J Otolaryngol Head Neck Surg. 2019;71(S3):1962-71. do:-10.1007/s $2070-018-1384-6$

21. Levinsen M, Kiilgaard JF, Thomsen C, Heegaard S, Nissen KR. Medical and surgical treatment of rhino-orbital-cerebral mucormycosis in a child with leukemia. Am J Ophthalmol Case Rep. 2021;22:101092. doi:10.1016/j.ajoc.2021.101094

22. Honavar SG, Sen M, Lahane S, Lahane TP, Parekh R. Mucor in a Viral Land: A Tale of Two Pathogens. Indian J Ophthalmol. 2021;69(2):244-52. do1:10.4103/1]0.1j0_3774_20.

23. Shah K, Dave V, Bradoo R, Shinde C, Prathibha M. Orbital Exenteration in Rhino-Orbito-Cerebral Mucormycosis: A Prospective Analytical Study with Scoring System. Indian J Otolaryngol Head Neck Surg. 2019;71(2):259-65. 101:101007/s 2070-(0)8-1293-8. 


\section{Author biography}

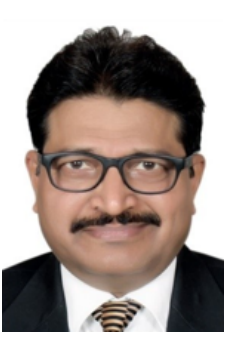

Rajendra Prakash Maurya, • Editor in Chief IP IJOOO,

- Associate Professor \& I/c Orbit, Ocular Oncology and Oculoplasty Unit Regional Institute of Ophthalmology, Institute of Medical Sciences, Banaras Hindu University, Varanasi, (UP), India

- Joint secretary Oculoplastics Society of South Asia.
E-mail:

mauryarp_bhu@yahoo.com

ditorijceo@gmail.com,

Cite this article: Maurya RP. Indications for orbital exenteration in COVID-19 associated Rhino-orbito-cerebral Mucormycosis. IP Int J Ocul Oncol Oculoplasty 2021;7(2):105-108. 